FRANZ, Heike, Zwischen Markt und Profession. Betriebswirte in Deutschland im Spannungsfeld von Bildungs- und Wirtschaftsbürgertum (1900-1945)

Hervé Joly

\title{
OpenEdition
}

Édition électronique

URL : http://journals.openedition.org/ifha/1283

DOI : $10.4000 /$ ifha. 1283

ISSN : 2198-8943

Éditeur

IFRA - Institut franco-allemand (sciences historiques et sociales)

Référence électronique

Hervé Joly, «FRANZ, Heike, Zwischen Markt und Profession. Betriebswirte in Deutschland im Spannungsfeld von Bildungs- und Wirtschaftsbürgertum (1900-1945)», Revue de l'IFHA [En ligne], Date de recension, mis en ligne le 01 janvier 2001, consulté le 22 septembre 2020. URL : http:// journals.openedition.org/ifha/1283; DOI : https://doi.org/10.4000/ifha.1283

Ce document a été généré automatiquement le 22 septembre 2020.

(C)IFHA 


\section{FRANZ, Heike, Zwischen Markt und Profession. Betriebswirte in Deutschland im Spannungsfeld von Bildungs- und Wirtschaftsbürgertum (1900-1945)}

Hervé Joly

Cet ouvrage, issu d'une thèse de doctorat soutenue à Bielefeld sous la direction de Peter Lundgreen, s'inscrit dans la longue liste des publications générées par le très fructueux programme de recherche spécial (Sonderforschungsbereich) « Bourgeoisie » mené dans les années 90 dans le cadre de cette université. Alors que la majorité des recherches portait sur le XIXe s., H.F. a abordé le terrain encore largement inexploré de la bourgeoisie allemande au XXe s. À travers l'étude de la constitution de la gestion (Betriebswirtschaft) en discipline universitaire, elle pose la question fondamentale de l'évolution de la relation entre la bourgeoisie d'affaires et la bourgeoisie de la Bildung. Elle montre que, de manière surprenante, la création des premières écoles supérieures de commerce, intervenue en Allemagne à partir de 1898, repose surtout sur l'initiative des enseignants des écoles secondaires commerciales qui se sont montrés désireux d'améliorer un statut jusqu'alors précaire par l'acquisition d'une formation supérieure, à l'image de leurs collègues des lycées classiques. L'enjeu est donc moins économique que social. Le développement des écoles supérieures de commerce se heurte d'ailleurs à une double opposition : d'une part, celle de nombreux entrepreneurs qui considèrent qu'une formation théorique est inutile pour l'exercice des fonctions commerciales et, d'autre part, celle des disciplines établies, et en particulier de l'économie politique (Volkswirtschaft), qui n'admettent pas que l'enseignement de cette "fausse science " que serait la gestion puisse relever d'une formation supérieure. Ce contexte initial pèse dans l'orientation prise par cette discipline en Allemagne. Elle doit à la fois démontrer son utilité auprès des praticiens en mettant l'accent sur les aspects les plus concrets, en particulier la comptabilité, tout en défendant une ambition théorique pour obtenir une reconnaissance universitaire perçue comme indispensable. 
2 Cette contradiction explique la réussite seulement imparfaite de la professionnalisation des gestionnaires. Ils ont bien obtenu en l'espace d'une trentaine d'années la consécration académique complète qu'ils recherchaient, couronnée en 1930 par l'attribution aux écoles supérieures de commerce du droit de délivrance du doctorat ; les diplômés ne connaissent guère, même dans les années 30, le souci du chômage mais ils ne sont pas parvenus à acquérir un monopole d'exercice dans des professions, à l'image des médecins ou des juristes, la période nazie également étudiée par H.F. n'apportant pas d'évolution majeure sur ce point. Dans les entreprises, leur accès aux responsabilités dirigeantes est resté, même dans les fonctions commerciales, limité, face aux multiples concurrences des ingénieurs, des juristes, des économistes et des praticiens passés par l'apprentissage interne. Ils sont surtout présents dans des postes plus modestes de comptables plus conformes à la réalité de leur formation. Dans l'administration, ils n'ont pas obtenu la remise en cause du monopole des juristes sur les emplois de la haute fonction publique. Même dans les professions libérales du contrôle de gestion et du conseil, auxquelles ils pouvaient apparaître particulièrement prédestinés, ils n'ont pas réussi à obtenir un accès exclusif en leur faveur.

3 Ce livre apporte donc une contribution importante à l'histoire de l'enseignement de la gestion en Allemagne, corrigeant la vision trop optimiste que l'historien américain Robert Locke avait donné, dans ses travaux comparatistes, de sa reconnaissance professionnelle. Même si H.F. n'a pas eu les moyens de mener les études empiriques qui seraient indispensables pour préciser les trajectoires des diplômés, elle contribue également à l'histoire de la bourgeoisie allemande, montrant de manière convaincante la prégnance persistante du modèle de la Bildung dans un secteur qui lui était pourtant a priori très éloigné.

Hervé JOLY 